\title{
Wearable Bio-Signal(PPG)-Based Personal Authentication Method Using Random Forest and Period Setting Considering the Feature of PPG Signals
}

\author{
Sun-Woo Lee ${ }^{1}$, Duk-Kyun Woo ${ }^{2}$, Yong-Ki Son ${ }^{3}$, Pyeong-Soo Mah² \\ ${ }^{1}$ Department of ICT, University of Science and Technology (UST) , Daejeon, Rep. of Korea. \\ ${ }^{2}$ Embedded System Software Research Group, Electronics and Telecommunications Research Institute \\ (ETRI). \\ ${ }^{3}$ Wearable Computing Research Institute, Electronics and Telecommunications Research Institute (ETRI). \\ * Corresponding author. Tel.: +82-42-860-6559; email: pmah@etri.re.kr \\ Manuscript submitted February 13, 2019; accepted April 15, 2019. \\ doi: 10.17706/jcp.14.4.283-294
}

\begin{abstract}
A study regarding personal authentication based on PPG has been conducted using the Random Forest algorithm. In order to ensure correct authentication, data features must be consistent. This consistency is provided through the normalization of the PPG signal using maximum-minimum normalization and spline interpolation. The threshold is set by using normalized data and the highest value among the points above the threshold is set as the peak of the period. After establishing candidates of valley with values below the threshold, a shifted period is calculated by designating the last valley next to the following peak to the valley of the period. In order to reduce errors in the one period PPG data, the data is averaged after overlapping. A discrete cosine transform (DCT) is used to extract features from the preprocessed data. Thus, the extracted features are used as the input variables for machine learning techniques such as Decision Tree, KNN, Random Forest. The accuracy of these algorithms is 93\%, 98\%, and $99 \%$ respectively.
\end{abstract}

Key words: Wearable, bio-signal, biometric authentication, machine learning, photoplethysmography (PPG).

\section{Introduction}

The development of information technology over the past few decades has brought about technological convenience, but the number of issues related to personal information, privacy invasion, and hacking is increasing. In order to solve this problem, password, PIN number, certificate, and biometric methods are used [1]. Unlike the other existing authentication methods, biometrics can be authenticated without the need to carry a certificate, they do not rely on memory, and they are difficult to duplicate.

Today's biometric authentication methods include fingerprint, iris, face recognition, electrocardiogram (ECG), and photo-plethysmography (PPG). The most prevalent among these methods is fingerprint recognition. However, in the case of this method, it can be difficult to confirm the identity of a fingerprint when the fingerprint is damaged. Moreover, there is the possibility of fingerprint falsification, as a fingerprint pattern can be reproduced using latex.

In the cases of ECG and PPG, reproduction is impossible since they rely on a bio-signal generated by the heartbeat. However, ECG requires at least two points of connection with the body, and there is the 
drawback of contamination problems that may occur due to issues related to electrode attachment.

In the case of PPG, the PPG sensor is attached to many wearable products such as many wearable watches and bands. Thus, it allows ease of measurement and there is little resistance to the measurement process. As an example, it is possible to provide increased convenience to people automatically opening/closing the door of a car by authenticating the user using a wearable band rather than a smart key. The main problem associated with PPG is that the shape of the PPG signal forms a smooth curve and there are no visible feature points, leading to difficulties in selecting feature points and feature extraction sections. Therefore, it is necessary to find a method that addresses this issue. Another complication relates to the tendency of the PPG signal that can be affected by motion noise.

Existing PPG-based personal authentication methods can be classified into two types: Fiducial (F) and Non-Fiducial (NF). The Fiducial method is an authentication approach that relies on finding the features of the signal in the time domain of the PPG signal. This process of finding the features in the time domain is conducted using two methods: using the PPG signal and Acceleration PPG (APG), typical signals of which are shown in Fig. 1. There have been several studies on the former approach and Y. Gu et al. introduced a personal authentication algorithm that uses the PPG signal [2], [3]. As a result, Y. Gu et al. obtained a 94\% authentication result through 17 PPG data signal features of the peak, time interval, upward slope, and downward slope [3]. The simplicity of the signal adds a disadvantage to the use of PPG signals since it is difficult to analyze and detect the change of phases. In order to compensate for this, previous studies have been conducted second derivatives of PPG signal in order to obtain features using APG signals [4]-[6].

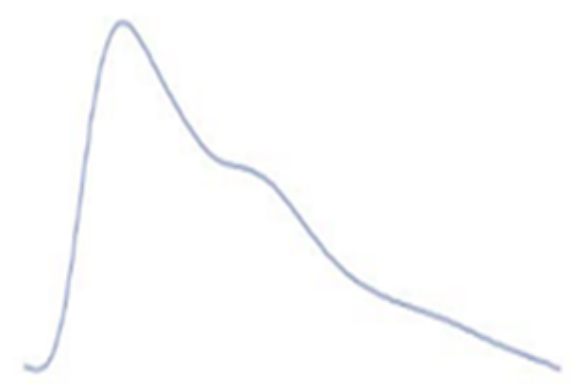

(a) PPG signal

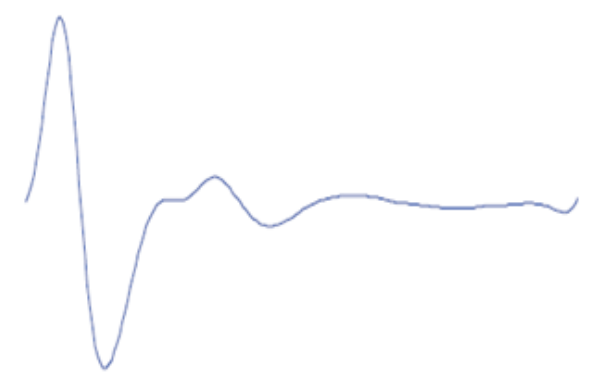

(b) APG signal

Fig. 1. Comparison of PPG and APG signal.

Jaafar et al. [5] used features extracted from APG signals in order to provide a 97.5\% result for personal authentication using a Bayes network and a K nearest neighbor classification technique.

Although most studies have been conducted using the fiducial method, Karimian et al. [7] obtained better results with a non-fiducial method using ECG data. However, in the case of one non-fiducial method, there is a difficulty in interpreting results obtained using data extracted using Discrete wavelet transforms (DWT) because it is expressed in the form of complex numbers [8]. 


\section{Proposed PPG-Based Biometric Authentication}

In this study, we propose a biometric authentication method using PPG signals with improved accuracy by complementing the disadvantages of existing PPG approaches due to the smooth shape of the PPG signal and the difficulties related to feature point and section selection. In order to improve accuracy, data features must be consistently preprocessed. To ensure data consistency, the peak is found in the PPG signal data, period normalization is then applied using the spline method, and the average is applied to the data that are overlapped three times. Discrete cosine transform (DCT) [9] is used through an extracting feature method and the Random Forest [10] machine learning method is applied as the personal authentication method.

As shown in Fig. 2, the proposed personal authentication method consists of several steps: preprocessing, feature extraction, identity verification, and conformation of results.

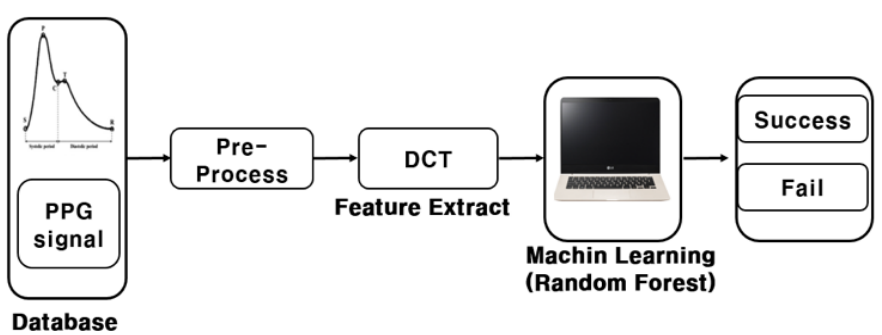

Fig. 2. Personal authentication procedure.

\subsection{Overview of Data Preprocessing Method}

The PPG signal is periodic, as an electrical signal that is affected by heartbeat. Some amount of DC component is to be removed from the PPG signal using Equation 1 to obtain an effective threshold. The closer the alpha value is set to 1 , the more DC component is removed. In this study, the DC component was removed by setting the alpha value to 0.95 recommended in [11].

$y(t)$ : output of the filter

$\mathrm{x}(\mathrm{t})$ : current input

$\mathrm{w}(\mathrm{t})$ : intermediate value

$\alpha$ : the response constant of the filter

$$
\begin{gathered}
\mathrm{w}(\mathrm{t})=x(t)+\alpha * w(t-1) \\
\mathrm{y}(\mathrm{t})=w(t)-w(t-1)
\end{gathered}
$$

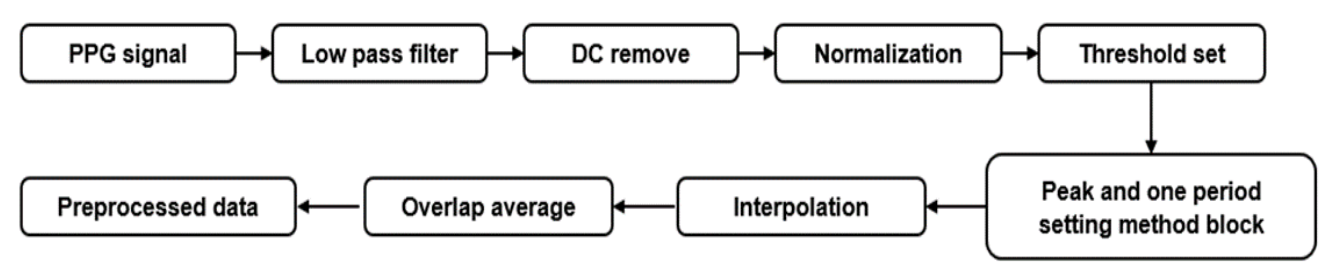

Fig. 3. Data preprocessing method.

Since the amplitude of each PPG signal is different for each individual, the magnitude of the amplitude of the PPG signal is normalized between 0 and 1, and maximum-minimum normalization relation given in Equation 2 is applied. 


$$
x_{\text {normalization }}[n]=\frac{x[n]-x_{\min }}{x_{\max }-x_{\min }}
$$

Using the normalized data, threshold is set by calculating an average using average filter which is shown in equation 3.

$$
\bar{x}_{k}=\frac{k}{k-1} \bar{x}_{k-1}+\frac{1}{k} x_{k}
$$

The normalized data and threshold are used for input of peak and one period setting method block. In order to reduce signal error, the data are interpolated and the average was set by preprocessing as shown in Fig. 3.

\subsection{Peak and Period Set Method}

Finding peak points in the PPG signal is a preliminary task to find out the periods. There are several methods to extract the peak, including utilizing zero-crossing method [12] and differentiating the PPG signal to set the largest change point as the peak [12]. In the study, the zero-crossing method is used to find out peaks in the PPG signal and the period is set by considering the peak-peak time. The threshold is calculated using the average filter shown in Equation 3 to extract the peak. Equation 3 improves the efficiency of calculation by applying the expression that uses only the previous mean result and the additional data instead of the batch expression that collects and calculates all the data at once.

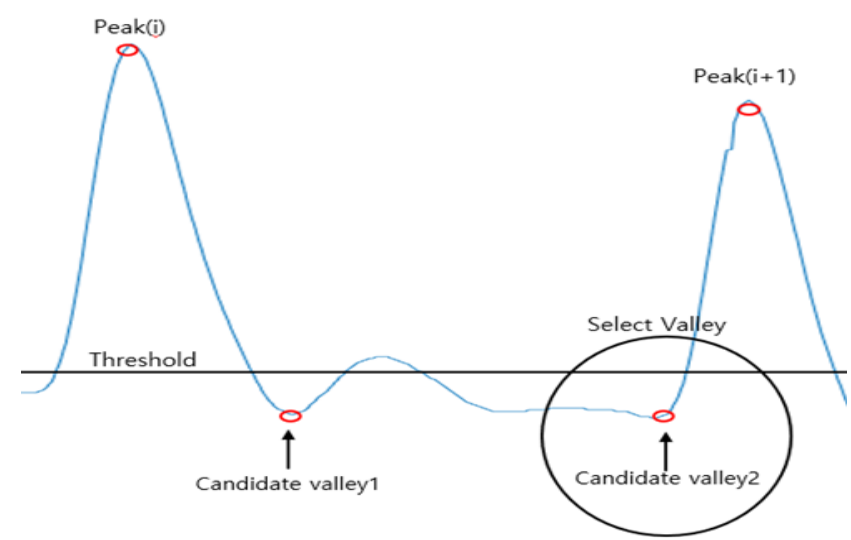

Fig. 4. period set method and valley set among the nominees.

As shown in Fig. 4, the Peak (i) is set as the component with the largest value above the threshold and peak $(i+1)$ is calculated by considering the peak-peak time. In order to obtain the characteristics of signals of subjects in one cycle of the PPG signal, the period is shifted to include components such as the systolic, diastolic peak and dicrotic notch. As a method to shift the period, the mean distance from the peak to valley is calculated and half of the mean distance is applied to shift. In the PPG signal, characteristics of these components are unique in each subject since these are produced when the heart is contracts and relaxes. In order to establish the period of signals which are affected by the contraction and relaxation of the heart, the setting point of the period is shifted to the left side of the peak. The smallest points which are below the threshold is set to the valley of the period. However, there can be a problem in case that there are many non-consecutive candidate valleys as shown in Fig. 4 . When the points that do not exceed the base point occur in more than two spots the last valley points that is the nearest valley to the next Peak, Peak(i+1) is 
chosen at the valley of the period. The block diagram of the above peak and period set method is shown in Fig. 5.

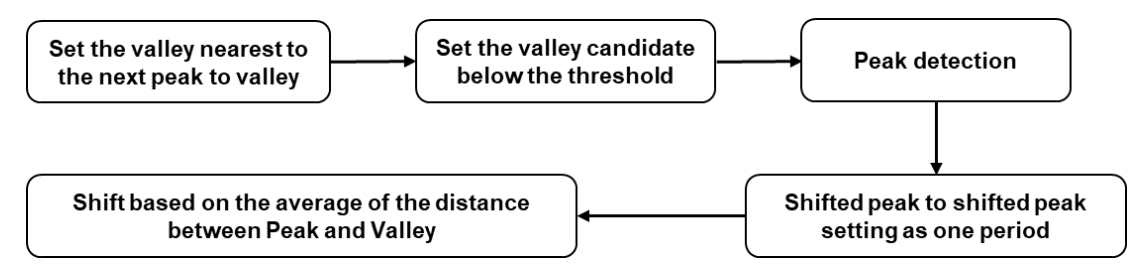

Fig. 5. A method of periodic extraction considering characteristics of PPG signal.

The PPG signal peak is extracted by considering periodicity and threshold. Unlike the approach employed in previous studies [13], the spline interpolation method is used in order to maintain the features of the PPG signal, instead of separating the fixed period length. It reduce the differences in the distance of one cycle and maintain data features.

The method used to set one period in the PPG data is given in Fig. 6. The average Peak-Valley distance is shifted in order to utilize the pulse features from Peak to Valley, instead of extracting features from signals between Shift_Peak Valley(SV)s. Thus, the data are preprocessed in order to express electronic features related to heartbeats in the PPG signals, setting up one cycle of the shifted SV.

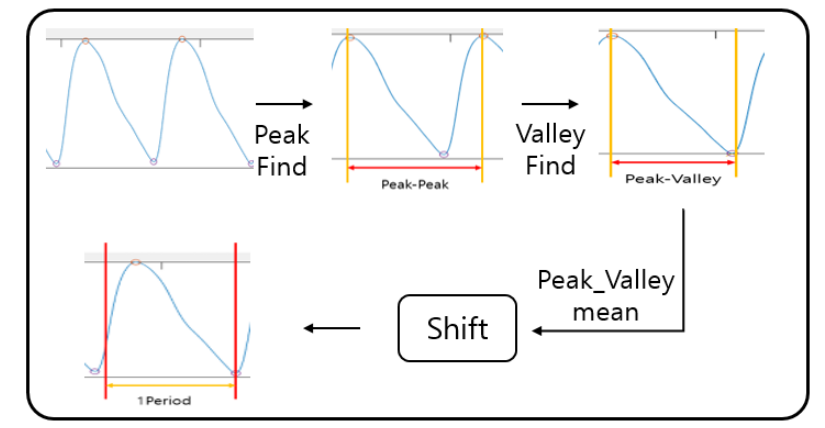

Fig. 6. Process of setting up one cycle of the PPG data.

\subsection{Error Reduction Method}

When averages are applied using an ensemble technique in order to extract individual features [14], issues related to machine learning training process may be introduced due to a reduction in the amount of data. As shown in Fig. 7, the data set is constructed here by overlapping three SV sections of one period in the PPG data, maximizing the total amount of data.

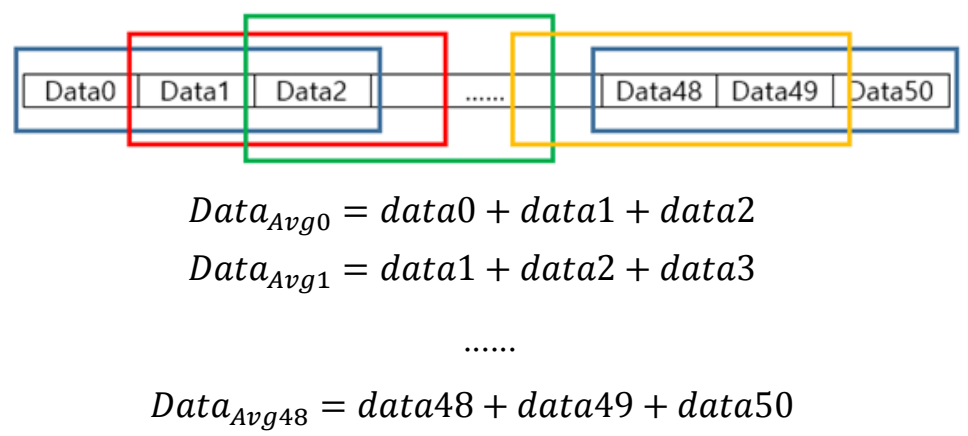

Fig. 7. Data averaging method. 
The SV interval is set up for one cycle and a specific feature is extracted using DCT for that cycle, and the extracted data contained in the $4-20 \mathrm{~Hz}$ range is used as that feature. In addition, if this feature is extracted using the DCT without using the overlapped average, errors will occur and reduce the accuracy of the personal authentication method. Overlapped averages are used in order to reduce error. A decrease in the difference between each cycle amplitude can be seen in Fig. 9, where the proposed overlapped average approach is used, compared to Fig. 8, where the original data is used.

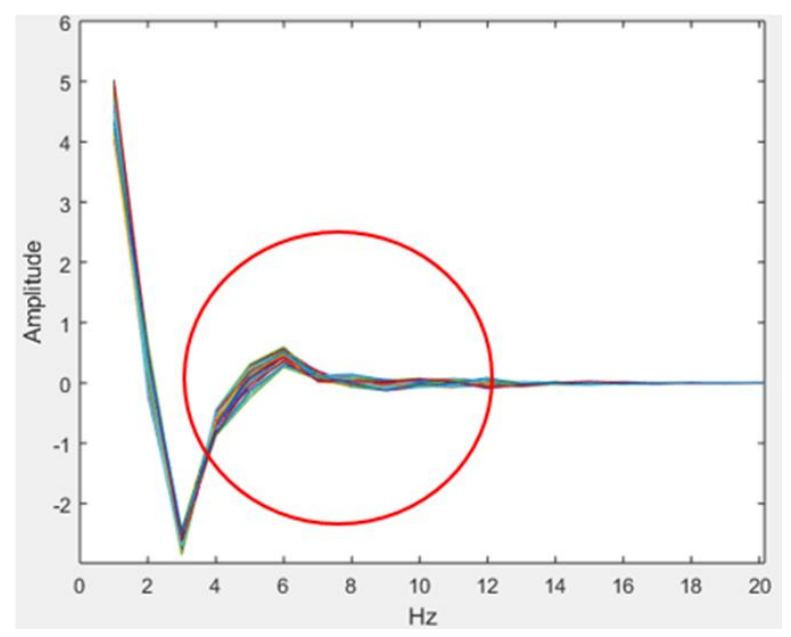

Fig. 8. DCT without overlap averaging.

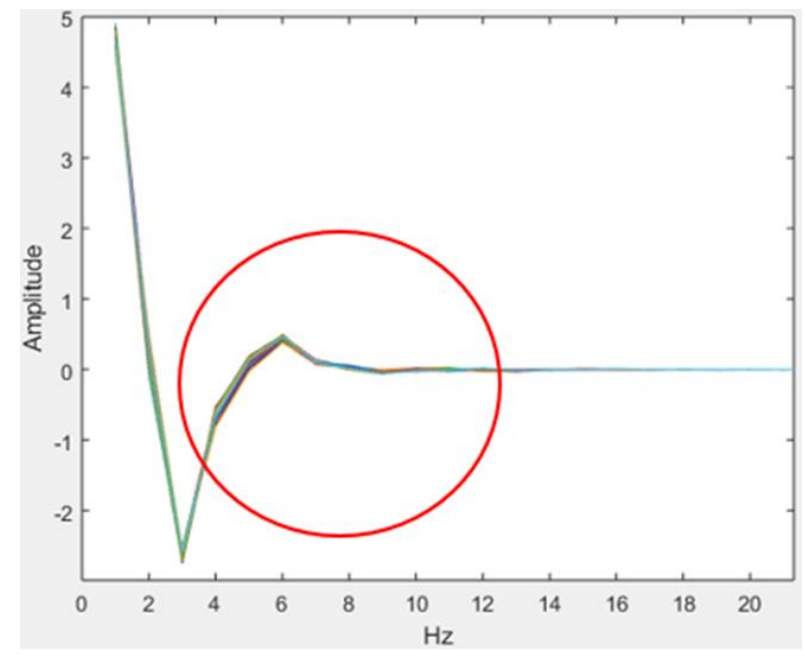

Fig. 9. DCT with overlap averaging.

\subsection{Feature Extraction Using Discrete Cosine Transform}

Since the individual features of the PPG data are located at low frequency, DCT is performed in order to extract low frequency features. In the case where using DWT produced results containing complex numbers [8], the necessary calculations are too complicated to be processed using wearable device. On the other hand, computational complexity is reduced by using DCT, which is a real-valued transformation method.

In previous studies [15], DCT was used as a feature extraction technique for ECG data which, like PPG, is one of the signals generated by heartbeat. Similar to the ECG data case, here the frequency components were concentrated at low frequencies in the PPG data. Here, DCT was successfully employed in extracting low-frequency features of the PPG data. 


\subsection{Personal Authentication Using Random Forest}

In order to conduct personal authentication, DCT related to the extracted data are used as input variables for the Random Forest process. The most important goal of the individual authentication process is to improve accuracy. Random Forest is better suited to personal recognition than other machine learning algorithms such as KNN [16] and SVM [16], because it is more accurate due to the capacity to change the number of standard trees that need to be identified.

The Random Forest machine learning algorithm was proposed by L. Reiman [10]. Fig. 10 shows the classification method of Random Forest. Random Forest is characterized by voting using Decision Trees [16].

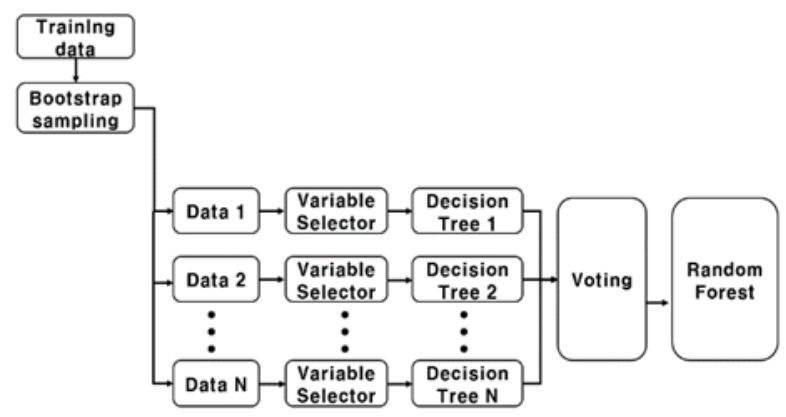

Fig. 10. Random forest classification method.

\section{Experiment and Results}

\subsection{Experimental Environment}

Experiments in this study were performed using the MATLAB and Python computer codes.

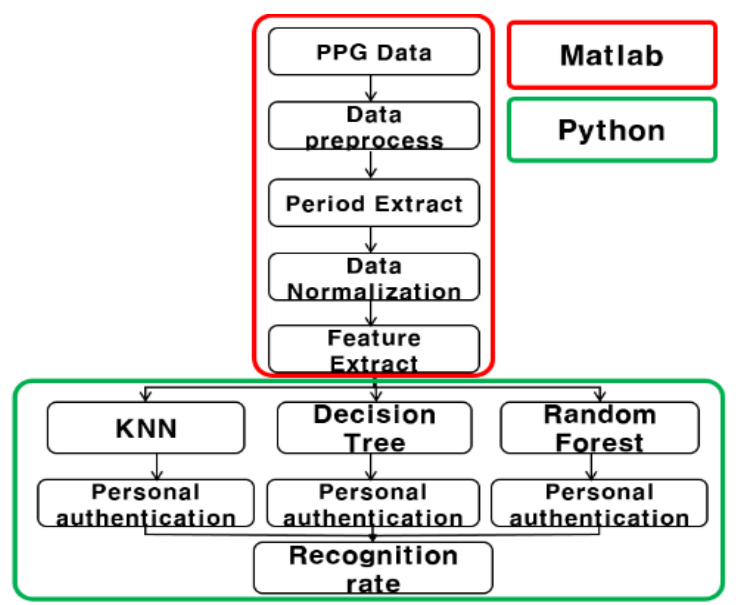

Fig. 11. PPG data processing environment.

TDME2013_PPG_Benchmark_R3 [17], [18] was followed, which is widely used for algorithm verification. The benchmark consists of 42 people, with a $300 \mathrm{~Hz}$ sampling rate and 8 minutes of data per person. Data were reconstructed using 100 periodic data within the 8-minute data time.

Table 1 shows the results of applying the Decision Tree (DT), KNN, and Random Forest (RF) machine learning techniques in order to reduce errors using both the proposed overlapped averages method and the original data without overlapped averages.

Using the proposed overlap average method, the accuracy of all machine learning techniques can be 
improved.

Table 1. Existing and Proposed Techniques

\begin{tabular}{|c|c|c|c|}
\hline AVG & $\begin{array}{l}\text { Decision } \\
\text { Tree }\end{array}$ & KNN & $\begin{array}{l}\text { Random } \\
\text { Forest }\end{array}$ \\
\hline Original data & $80 \%$ & $92 \%$ & $92 \%$ \\
\hline $\begin{array}{l}\text { After applying } \\
\text { overlap } \\
\text { average }\end{array}$ & $89 \%$ & $94 \%$ & $96 \%$ \\
\hline $\begin{array}{l}\text { Period and DCT } \\
\text { value overlap } \\
\text { average }\end{array}$ & $93 \%$ & $98 \%$ & $99 \%$ \\
\hline
\end{tabular}

\subsection{Comparison of Accuracy Based on Amount of Data}

To use PPG signals for authentication in real environment, it should not take too long and maintain accuracy. If it takes a long time, user may feel uncomfortable. Therefore, we experimented to see how accuracy and time vary as we change the number of pulse. Table 2 shows the accuracy of each machine learning method using $10,30,50$, and 100 peak periods. The accuracy of individual authentication varies according to the amount of data that is presented for machine learning.

Table 2. Existing and Proposed Preprocessing Techniques

\begin{tabular}{llll}
\hline Periods & $\begin{array}{l}\text { Decision } \\
\text { Tree }\end{array}$ & KNN & $\begin{array}{l}\text { Random } \\
\text { Forest }\end{array}$ \\
\hline 10 & $87 \%$ & $96 \%$ & $98 \%$ \\
30 & $91 \%$ & $98 \%$ & $99 \%$ \\
50 & $94 \%$ & $98 \%$ & $99 \%$ \\
100 & $93 \%$ & $98 \%$ & $99 \%$ \\
\hline \hline
\end{tabular}

Decision Tree showed the greatest change in accuracy according to the amount of data. In the case of Random Forest, performance was consistent above 30 periods of data. Also, the larger periods are used, the faster the result of experiment, with the same accuracy.

\subsection{Setting Optimal Parameters for Machine Learning}

In order to extract the features of each pulse in the data, DCT was performed to obtain DCT coefficients. Pulses were extracted within the range from 4 to $20 \mathrm{~Hz}$, as the features required to distinguish individuals are distributed at frequencies below $20 \mathrm{~Hz}$.

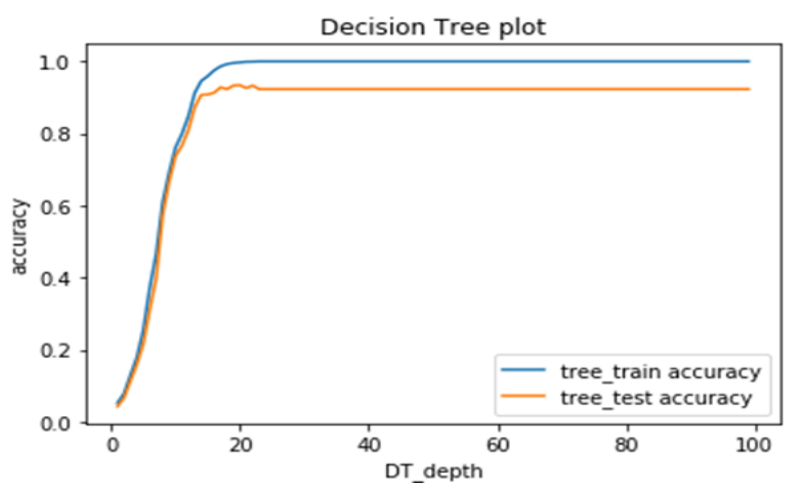

Fig. 12. Decision tree experiment results. 


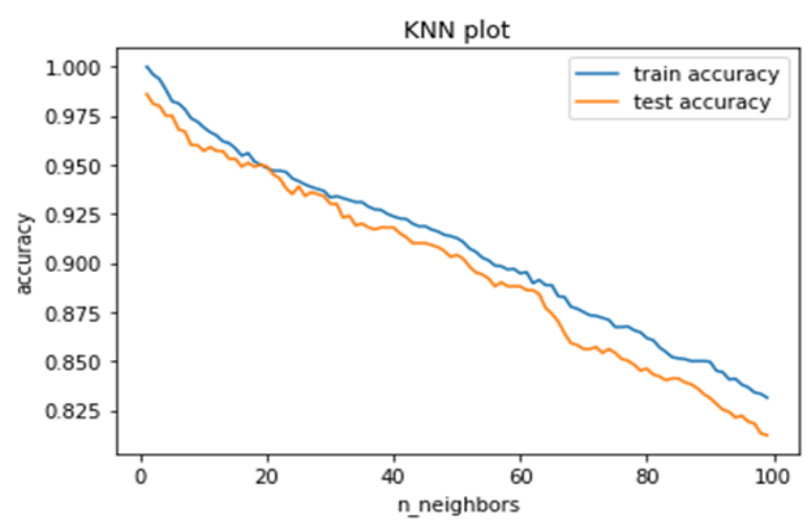

Fig. 13. KNN experiment results.

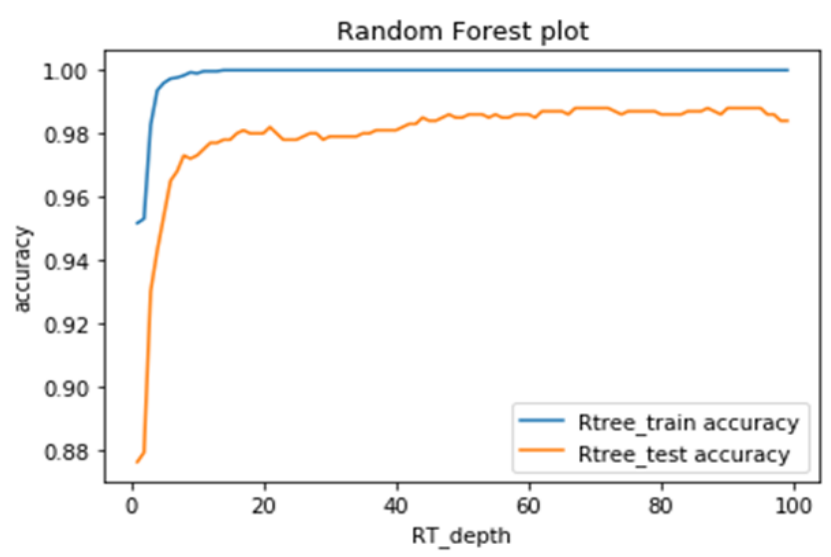

Fig. 14. Random forest experiment results.

Features were extracted from the DCT of PPG data and the performances were verified using DT, KNN, and RF techniques. In order to compare the performance of each machine learning algorithm, the accuracy of the algorithm is measured by increasing the parameters of the algorithm. The results for each machine learning algorithm are shown in Fig. 12, 13, and 14.

In the case of the DT method, the optimal number of trees is determined by specifying the number of optimal trees, the number of neighbor's $\mathrm{K}$ is specified for KNN, and the number of trees is chosen for the Random Forest method.

For the Decision Tree method, the optimal depth parameter was chosen at a tree depth of 21, after which there is no further increase in accuracy. In the case of KNN, the model is simple and the accuracy is lowered as $\mathrm{K}$ increases. In the case of the Random Forest method, the optimal parameters were obtained using 66 trees, after which the results were stable and no further increase in accuracy is obtained.

Following these tests, the DT experiment was carried out using a depth parameter of 19, yielding an accuracy result of $93 \%$. In the case of KNN, the accuracy result was $93 \%$ at $\mathrm{K}=2$. In the case of Random Forest, the accuracy was $99 \%$ when using 66 trees. The proposed authentication method may be compared with existing authentication methods that employ PPG data.

In the case of Gu et al. [2], [3], which describes the use of PPG signals in the literature for the first time, four features were extracted from the PPG signal, experiments were performed on 17 individuals, yielding an accuracy result of $94 \%$. However, more research is needed to investigate how to use a small amount of data in a real environment, since it may be difficult to extract features in case PPG signals are smooth.

In order to solve this problem, Sammik [4] and Jaagar [5] used APG signals in experiments with 15 and 
10 people, respectively, using the Fiducial method, and obtained accuracy results of $100 \%$ and $97.5 \%$, respectively. However, since these data sets were small, further research is required in a real environment. In addition, the fiducial method using APG signals can be subject to issues related to the features such as the Dicrotic notch (see Fig. 15 c, d), which cannot be properly extracted from APG data when the inflection point is smooth.

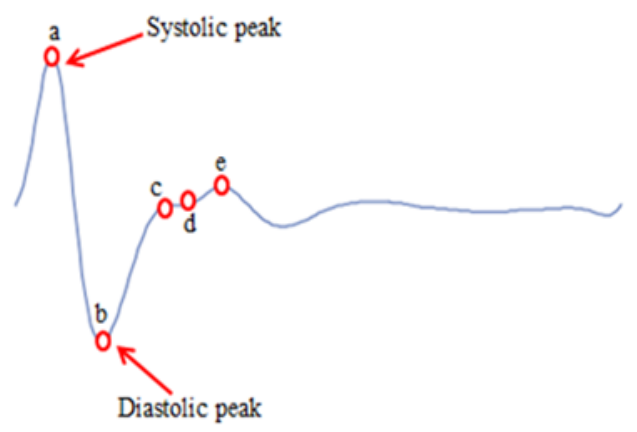

Fig. 15. Feature points of APG data.

However, the proposed method provides lower errors than the APG feature extraction method because our proposed method uses only the peak and valley in the PPG signal is used.

In the case of the Non-Fiducial method, Karimian [8] obtained a result of $100 \%$ for individual authentication, although the use of DWT as a feature extractor may mean that the calculations are too complicated for a wearable device environment.

The proposed method, however, uses DCT in order to simplify the calculation process in a wearable device environment, and when using Random Forest, 42 people were authenticated with an accuracy of 99\%. Therefore, a combination of Random Forest and DCT is suitable for use with wearable devices.

\begin{tabular}{|c|c|c|c|}
\hline Study & $\begin{array}{l}\text { Feature } \\
\text { (Subject) }\end{array}$ & $\begin{array}{l}\text { Classification } \\
\text { Method }\end{array}$ & Accuracy \\
\hline $\begin{array}{c}\mathrm{Gu} \\
\text { et al[2][3] }\end{array}$ & $\begin{array}{c}\text { Fiducial } \\
\text { (17) }\end{array}$ & $\begin{array}{c}\text { Euclidean } \\
\text { Distance [2] } \\
\text { Fuzzy[3] }\end{array}$ & $94 \%$ \\
\hline $\begin{array}{l}\text { Chakrabory } \\
\text { et al }[4]\end{array}$ & $\begin{array}{c}\text { Fiducial } \\
\text { (15) }\end{array}$ & LDA & $100 \%$ \\
\hline $\begin{array}{c}\text { Jaagar } \\
\text { et al[5] }\end{array}$ & $\begin{array}{l}\text { Fiducial } \\
\text { (10) }\end{array}$ & Bayes Network & $97.5 \%$ \\
\hline $\begin{array}{c}\text { Kavsoglu } \\
\text { et al[6] }\end{array}$ & $\begin{array}{l}\text { Fiducial } \\
\qquad(40)\end{array}$ & KNN & $94.4 \%$ \\
\hline $\begin{array}{c}\text { Karimian } \\
\text { et al[8] }\end{array}$ & $\begin{array}{c}\text { Non-Fiducial } \\
\quad(42)\end{array}$ & $\mathrm{GA}+\mathrm{ANN}$ & $100 \%$ \\
\hline Proposed & $\begin{array}{c}\text { Non- Fiducial } \\
\text { (42) }\end{array}$ & Random Forest & $99 \%$ \\
\hline
\end{tabular}

Table 3 summarizes results obtained using several authentication methods by various researchers, including the Non-Fiducial method using PPG and APG and other existing fiducial methods.

\section{Conclusion and Future Work}

In the proposed individual authentication method, machine learning approach was used together with spline interpolation and overlap averaging. The accuracies of decision tree, KNN, and Random Forest were $93 \%, 98 \%$, and $99 \%$, respectively. 
In the case of personal authentication using PPG data, the accuracy of personal authentication is affected by the method of preprocessing the data. A shifted period can be controlled by selecting the last candidate valley as the valley of the period, even though the size of PPG signal. Periodic normalization is performed using spline interpolation and data is preprocessed using maximum-minimum normalization. DCT is employed in order to reduce computational complexity and to take advantage of the convergence of signals with features that distinguish a person at low frequency in the PPG signal. As a result of applying machine learning approach with extracted features, the best performance of $99 \%$ was provided by the Random Forest method.

However, at least 66 trees are needed during the training process, which causes issues related to computational speed. The advantage of proposed method, on the other hand, is that it may be applied to wearable bands that are always worn, as automobile key alternatives that can authenticate users and allow the automatic opening and closing of doors.

In the future, it is necessary to apply this approach to much larger sets of individuals and examine the resulting data and identification accuracy. Another important factor will be to study the robustness of this wearable technology with respect to noise, which can be applied in an embedded environment.

\section{Acknowledgment}

This work was supported by Korea Evaluation Institute of Industrial Technology (KEIT) grant funded by the Korea government (MOTIE) (No.10065738, Development of wearable development toolkit for various wearable application services).

\section{References}

[1] Korea Institute of Science and Technology information (KISTI) MARKET REPORT, 48, 2016.

[2] Gu, Y. Y., Zhang, Y., \& Zhang, Y. T. (2003). A novel biometric approach in human verification by photoplethysmographic signals. Proceedings of 4th International IEEE EMBS Special Topic Conference on Information Technology Applications in Biomedicine (pp. 13-14).

[3] Gu, Y. Y., \& Zhang, Y. T. (2003). Photoplethysmographic authentication through fuzzy logic. Proceedings of IEEE EMBS Asian-Pacific Conference on Biomedical Engineering (pp. 136-137).

[4] Chakraborty, S., \& Pal, S. (2016). Photoplethysmogram signal based biometric recognition using linear discriminant classifier. Proceedings of 2016 2nd International Conference on Control, Instrumentation, Energy \& Communication (CIEC) (pp. 183-187).

[5] Jaafar, N. A. L., Sidek, K. A., \& Azam, S. N. A. M. (2015). Acceleration plethysmogram based biometric identification. Proceedings of 2015 International Conference on BioSignal Analysis, Processing and Systems (ICBAPS) (pp. 16-21).

[6] Kavsaoğlu, A. R., Polat, K., \& Bozkurt, M. R. (2014). A novel feature ranking algorithm for biometric recognition with PPG signals. Computers in Biology and Medicine, 49, 1-14.

[7] Karimian, N., Guo, Z., Tehranipoor, M., \& Forte, D. (2017). Highly reliable key generation from electrocardiogram (ecg). IEEE Transactions on Biomedical Engineering, 64(6), 1400-1411.

[8] Karimian, N., Guo, Z., Tehranipoor, M., \& Forte, D. (2017). Human recognition from photoplethysmography (ppg) based on non-fiducial features. Proceedings of 2017 IEEE International Conference on Acoustics, Speech and Signal Processing (ICASSP) (pp. 4636-4640).

[9] Ahmed, N., Natarajan, T., \& Rao, K. R. (1974). Discrete cosine transform. IEEE Transactions on Computers, 100(1), 90-93.

[10] Chen, C., Liaw, A., \& Breiman, L. (2004). Using random forest to learn imbalanced data. University of California, Berkeley, 110, 1-12.

[11] Implementing pulse oximeter using MAX30100. Retrieved from 
https://morf.lv/implementing-pulse-oximeter-using-max30100

[12] Sarkar, A., Abbott, A. L., \& Doerzaph, Z. (2016). Biometric authentication using photoplethysmography signals. Proceedings of 2016 IEEE 8th International Conference on Biometrics Theory, Applications and Systems (BTAS) (pp. 1-7).

[13] Spachos, P., Gao, J., \& Hatzinakos, D. (2011). Feasibility study of photoplethysmographic signals for biometric identification. Proceedings of 2011 17th International Conference on Digital Signal Processing (DSP) (pp. 1-5).

[14] Choudhary, T., \& Manikandan, M. S. (2016). Robust photoplethysmographic (PPG) based biometric authentication for wireless body area networks and m-health applications. Proceedings of 2016 Twenty Second National Conference on Communication (NCC) (pp. 1-6).

[15] Jeong, K. K., Kang, B. L., \& Sang, G. H.(2017). ECG-based biometric authenticaion using random forest. Journal of the Institutue of Eletronics and Information Engineers, 54(6), 100-105.

[16] Blasco, J., Chen, T. M., Tapiador, J., \& Peris-Lopez, P. (2016). A survey of wearable biometric recognition systems. ACM Computing Surveys (CSUR), 49(3), 43.

[17] Karlen, W., Raman, S., Ansermino, J. M., \& Dumont, G. A. (2013). Multiparameter respiratory rate estimation from the photoplethysmogram. IEEE Transactions on Biomedical Engineering, 60(7), 1946-1953.

[18] Download the IEEE TBME respiratory rate benchmark data set. Retrieved from http://www.capnobase.org/database/pulse-oximeter-ieee-tbme-benchmark

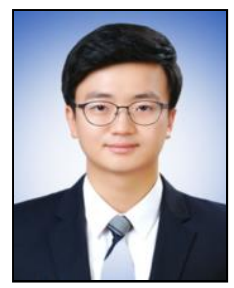

Sun-Woo Lee received the B.S degree in electronic engineering from the Daejeon University, Korea in 2016. He is currently studying for a master's degree in Department of ICT in computer software, University of Science and Technology (UST). His research interests are signal processing, wearable device, machine learning and embedded system.

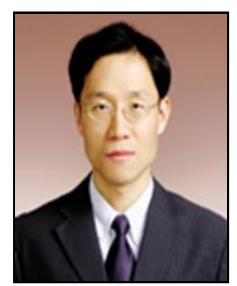

Duk-Kyun Woo received the BS, MS, and Ph.D degrees in computer science from Hongik University, Korea in 1993, 1995 and 2001. In 2001, he joined in the Embedded System Research Group at the Electronics and Telecommunications Research Institute (ETRI), Rep. of Korea, where he is currently a project leader and principal researcher. His research interests include embedded development solutions for wearable and IoT, instruction-level simulators, and compilers.

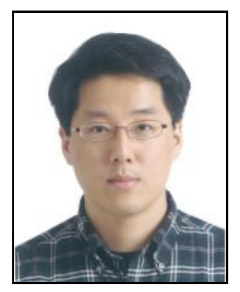

Yong-Ki Son received the B.S and M.S. degree in electrical and electronics engineering from Chung-ang University, Korea in 1999, and 2001. Since 2001, he has been a principal researcher of Electronics and Telecommunications Research Institute (ETRI). His research interests include human augmentation, wearable computer and human-computer interaction.

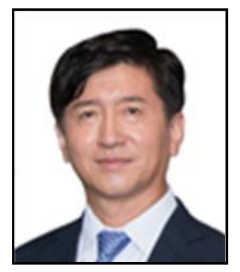

Pyeong-Soo Mah received the M.S and Ph.D degree in computer science and engineering from City University of New York, USA in 1992 and Wright State University, USA in 1995, respectively. Since 1996, he has been a principal researcher of Electronics and Telecommunications Research Institute (ETRI). His research interests include lightweight operating system, embedded software, and IoT systems. 